\title{
Introducción de innovaciones en la práctica clínica y capacitación médica. Comentario en relación a la reciente incorporación de la enfermedad vascular cerebral isquémica aguda, en el Régimen de Garantías Explícitas en Salud - Auge Adoption of clinical practice innovation and medical education. The case of the introduction of stroke as part of a national regimen of health guarantees
}

Jorge Nogales-Gaete ${ }^{1}$

To introduce innovation in clinical practice is a difficult task. Although there is no universal recipe, education of involved actors has been identified as a key factor, including managers, health care workers, patients, and community. Managers in charge of developing and implementing new clinical guidelines bear great responsibility, and need formal education on the appraisal of evidence and the analysis of available resources, to ensure the quality of the product that will be delivered. Although continuous education and training is widely perceived as valuableactivities, peopleneed to beencouraged to participatein them. Incentivesfor fostering participation are needed, and they should be carefully planned. The contents of the training programs should betailored to the different audiences and they should be delivered in attractive manner, in small groups, using modern interactive educational technology.

Key words: M edical education, continuing medical education, cerebrovascular disease. Palabras clave: Educación médica, educación médica continua, enfermedad vascular cerebral, programa Auge- GES.

Rev Chil Neuro-Psiquiat 2006; 44(4): 242-248

1 Servicio de Neurología Hospital Barros Luco, Departamento de Neurología

Sur. Facultad de Medicina Universidad de Chile. 


\section{Introducción}

El Sr. Editor me solicitó un comentario editorial, sobre la importancia de la capacitación médica en la situación de incorporación de innovaciones o cambios en la práctica clínica. Esto en relación a la situación generada por la incorporación de la enfermedad vascular cerebral (ECV) isquémica aguda, o accidente cerebrovascular (ACV) en el Régimen de Garantías Explícitas en Salud (GES)-Auge ${ }^{(1)}$.

Esta reflexión, se basa en nuestras diversas experiencias y en la revisión y análisis de experiencias publicadas. Admito, el potencial sesgo de análisis que podría determinar mi desempeño profesional como médico clínico especialista en neurología, con responsabilidad asistencial principalmente pública, en el Servicio de N eurología del Complejo Asistencial Barros Luco y labor docente universitaria en el Departamento de Neurología Sur de la Facultad de M edicina de la Universidad de Chile.

En relación específica al problema en comento, ACV-GES, en nuestro Servicio-Departamento hemos tenido que realizar comunitariamente, en forma local y autogestionada, el diseño e implementación de diversas actividades de capacitación ad-hoc fundamentalmente clínicas, en ausencia de información adicional a la disponible en la "guía clínica ACV-GES"(1). Las actividades listadas a continuación sustentan nuestra experiencia: reuniones clínicas, elaboración de guías de practica clínica, perfeccionamiento formal de personal de enfermería y técnicos paramédicos, generación de un programa de educación de enfermería para cuidadores y familiares de pacientes hospitalizados con ECV, visitas de capacitación a otros centros del país y extranjero de miembros del staff médico, organización de un ciclo de video conferencias nacionales sobre aspectos de la atención aguda de la ECV y condiciones necesarias para la implementación de trombolisis en el sector público con participación de neurólogos desde Arica a Punta Arenas, programa de capacitación para médicos con responsabilidad de gestionar interconsultas desde los policlínicos de atención primaria del territorio del SSMS a nuestro centro de diagnostico y tratamiento (CDT), programa de capacitación para médicos y enfermeras de los servicios de atención primaria de urgencia (SAPU) del SSM S y finalmente un programa de educación continua de neurología (incluyendo la ECV), a distancia, para médicos generales del país, a través de la plataforma de Internet M edichi de la Universidad de Chile. Todas estas modalidades de capacitación han determinado beneficio, pero este ha sido insuficiente para lograr fluidez en la atención de pacientes con ACV en acuerdo al sistema GES. Esta situación, podría deberse en grado diverso y combinado a falencias en nuestra organización, a insuficiencias en las tareas de información-capacitación, a falta de recursos y como ya ha sido planteado en una reciente publicación, a algunos problemas de la "guía clínica de ACV isquémico en el programa GES"(2).

El objetivo central de una capacitación, exigible al nivel gestor e impulsor de una innovación, en esta área y situación, es el dar a conocer el nuevo modelo de atención, su sustento, potenciales beneficios en los resultados, los recursos disponibles y la forma de implementación, lo que en este caso no ha ocurrido. Esto, a su vez con el propósito de facilitar el cambio de la práctica de atención, para generar una mejoría en los resultados de la atención sanitaria en el individuo y en la red de atención, lo que representa sin duda, el mayor desafío propio de una capacitación médica(3,4).

Cómo hacer la capacitación con esta mirada, con la mayor eficacia y eficiencia, en este particular paradigma, aquí y ahora, es algo aún no resuelto. Coincidimos con quienes 
piensan que para introducir una innovación o cambio en una práctica clínica, no hay recetas universales ${ }^{(5)}$. Por esto lejos de intentar dar alguna receta, nos hemos fijado como modesto propósito seleccionar y poner a disposición de los lectores, algunos insumos, en forma de preguntas, antecedentes y comentarios, para contribuir a la discusión en futuras iniciativas al respecto. ¿Es necesaria y suficiente la calidad de la innovación, para predecir su aceptación?, ¿Cómo motivar la capacitación e implementación de una innovación? ¿Funcionan la "evidencia" y el "Benchmarking", por sobre un determinado contexto?, estos son los aspectos que intentaremos comentar. Entendemos el Benchmarking como aquella técnica de gestión, que busca descubrir y definir los aspectos que hacen que una determinada empresa sea más eficiente y rentable que otra, para después intentar replicar o adaptar el conocimiento adquirido en otra empresa.

\section{Preguntas, antecedentes y comentarios}

1.- Si la innovación a implementar, en lo clínico y administrativo, explicitado en las guías de práctica clínica, fuesen adecuados en: sus potenciales beneficios en los resultados, sustento en cuanto a evidencia técnica y respaldo de recursos, para su implementación, lo que como vimos en este caso, en la actualidad es discutible ¿sería suficiente para el predecir el éxito de la capacitación?

Antecedentes: Un estudio basado en una encuesta realizada a 754 médicos generales o de atención primaria, de Francia, Alemania, Reino Unido, Italia y Suecia en torno a la implementación de una guía europea para la prevención primaria de enfermedad coronaria $^{(6)}$, nos puede orientar al respecto.

En este estudio el $91 \%$ de los profesionales encontraba útil la guía, $89 \%$ estaban de acuerdo en algún grado con el contenido ( $29 \%$ en forma total y $60 \%$ en forma parcial), $87 \%$ consideraron que la guía respondía a una necesidad, el $81 \%$ al momento de incorporar la guía ya usaba una.

A pesar de una organizada y sostenida campaña de promoción para el uso de esta guía, solo el $15 \%$ la usaba, el $12 \%$ reconocía no conocerla y un $19 \%$ no usaba ninguna guía. Sólo el $18 \%$ de los encuestados consideraba que la guía finalmente sería bien implementada. Los problemas mencionados por el resto, como limitantes para la implementación fueron: 38\% la consideraba como una causa de pérdida de tiempo adicional, $30 \%$ por los costos asociados y $17 \%$ por la limitada proyección de adherencia de los pacientes. Como sugerencias para mejorar la implementación fueron planteadas las siguientes medidas: educación a mé dicos $29 \%$, educación a pacientes $25 \%$, promoción y marketing general $23 \%$, simplificando la guía $17 \%$ y haciéndola más clara 12\%. Las formas de capacitación consideradas como favorecedoras de buenos resultados son las: comunitarias, en grupo pequeño, directas, participativas, coherentes con los cambios organizacionales pertinentes y asociados a incentivos por buena práctica, más que penalización por omisión.

Los componentes identificados en general, como favorecedores para la implementación de una guía de esta naturaleza son: adecuada identificación de las llaves de opción y sus consecuencias en el desenlace clínico, clara evidencia del beneficio clínico y de la ventaja en el costo de decisiones alternativas e implementación práctica lo mas simple posible.

Al menos dos objeciones podrían hacerse de este estudio, la primera es el alto índice de rechazo a la entrevista, 47 a 83\%, en los diferentes países, por lo que los 754 médicos que efectivamente contestaron podrían tener el sesgo de ser los mas voluntariosos y dispuestos a este tipo de investigaciones, y la segunda es que entre el decir (en una encuesta) y el hacer 
(en la práctica) muchas veces hay un largo trecho. La significación estadística del instrumento fue establecida como de un $95 \%$, con un $5 \%$ de nivel de riesgo.

Comentario: Aún una guía considerada como necesaria, útil, pertinente en contenido y sustento de este, con beneficios en los resultados y con respaldo de recursos para su implementación, tiene dificultades en su incorporación como nueva prácti$\mathrm{ca}$, en un grupo favorable para estos instrumentos. No existen estudios sobre que pasa con la implementación de las nuevas prácticas, que no cumplen con estos atributos técnicos y respaldos de recursos.

2.- Si la calidad del producto o innovación a implementar es una condición necesaria, pero no suficiente ¿Existen motivaciones o estímulos para mejorar
Tabla 1. Estímulos para mejorar la efectividad del aprendizaje médico

\begin{tabular}{lc}
\hline Estímulo & $\begin{array}{r}\text { \% de artículos } \\
\text { que mencionan } \\
\text { este estímulo }\end{array}$ \\
\hline Retroalimentación & 39 \\
Reiteración & 25 \\
$\begin{array}{ll}\text { Cursos con reconocimiento curricular } \\
\text { formal como EM C o postítulo universitario }\end{array}$ & 14 \\
$\begin{array}{ll}\text { Existencia dediversosniveles decomplejidad } \\
\text { Entrega con estrategias docentes múltiples }\end{array}$ & 10 \\
$\begin{array}{l}\text { Amplitud de espectro dela cobertura delas } \\
\text { situaciones clínicas planteadas }\end{array}$ & 9 \\
$\begin{array}{l}\text { Participación directa y activa en el proceso } \\
\text { docente }\end{array}$ & 9 \\
Objetivos claros del curso & 6 \\
Validez delas simulaciones empleadas & 3 \\
\hline
\end{tabular}

la efectividad del aprendizaje médico y su producto, el cambio de conducta?

La medicina basada en la evidencia ha influido con su esencia y marco metodológico(7), a otros dominios del conocimiento y análisis, como la educación médica. Por eso, elegimos como insumo para esta respuesta una revisión sistemática elaborada con metodología de nominada "educación médica basada en la mejor evidencia"(4).

Antecedentes: La tabla 1 muestra algunos estímulos reconocidos como útiles para mejorar la efectividad y producto del aprendizaje médico. Aun cuando el origen de esta revisión, que abarca 109 publicaciones realizadas entre 1969 y 2003, alude a lograr aprendizaje efectivo usando metodologías de simulación ${ }^{(8)}$, los estímulos mencionados pueden ser considerados como generales a la educación médica.

Comentario: Los elementos aludidos sugieren expectativas y valoración, de quienes se capacitan, por estrategias docentes bien planificadas, con diversas herramientas y niveles de enseñanza, que consideran en su diseño y desarrollo las características y necesidades del usuario, y que además son continuas y reiteradas y finalmente gestionadas y certificadas por instituciones formales de educación.

3.- ¿Que otros elementos han sido mencionados, en publicaciones especializadas, como favorecedores de las prácticas de educación médica continua y cambios de conducta, en escenarios como este?

Antecedentes: En este aspecto, el listado de elementos y sugerencias, respaldados con variado grado de sustento y evidencia, es extenso y diverso, por lo que seleccionaremos solo algunos de ellos.

La actitud y accionar de la organización donde se desempeña el alumno, en términos de tener una conducta coherente, facilitadora y reconocedora de la capacitación y el logro del 
cambio, es otro elemento considerado como favorecedor $r^{(3)}$.

La auto identificación de la necesidad del cambio por los alumnos, generada por inquietudes de pacientes, de colegas y malas respuestas a tratamiento, aparece como importan$\mathrm{te}^{(9,10)}$.

Una capacitación que da cuenta de la realidad organizacional y de la práctica clínica real del alumno, es mejor valorada(11).

La concordancia entre el contenido de la innovación y los cambios organizaciones necesarios para implementarla, son básicos ${ }^{(11)}$.

Respecto de la modalidad de enseñanza, existe acuerdo en la validez complementaria de la diversidad de instrumentos de docencia en oferta ${ }^{(12)}$, así como existe controversia respecto de reconocer, si la modalidad de seminarios y clases clásicas o los modelos basados en enseñanza centrada en casos y problemas tienen ventajas significativas para una opción universal(13-16).

Comentario: Las razones que influyen en los cambios de práctica clínica, son complejas y diversas como se señala en un artículo, en una sección temáticamente homologable, que discute las influencias que reconoce un médico para prescribir un nuevo fármaco(17). El tipo de información (publicación, conferencia, marketing), la acreditación de la autoridad que la entrega, el análisis en reuniones clínicas, la opinión de especialistas, la interacción con colegas pares, las guías clínicas, los resultados clínicos observados, la importancia de los efectos secundarios, el costo del fármaco, las indicaciones de su hospital, la información aportada por los laboratorios, son todos considerados como influyentes en forma integrada, aunque ningún elemento aislado aparece claramente como el determinante en la decisión final del médico ${ }^{(17)}$. Esta complejidad debe ser asumida por los directivos y gestores que propician un cambio, una guía, un instructivo o un decreto, no bastan.

4.- ¿Y parafraseando la técnica del Benchmarking, será cosa de buscar la mejor evidencia en educación para luego replicarla en nuestro programa de capacitación en ACV, en función del marco GES, en Chile?

Antecedentes: En un interesante artículo sobre las lecciones que la medicina basada en evidencia (MBE) podría proporcionar a la "educación médica basada en la mejor evidencia" ${ }^{(18)}$, podemos encontrar algunas orientaciones.

En este artículo se comenta sobre la dificultad que significa realizar la revisión y síntesis de la evidencia, sobre el aporte de la cooperación y voluntarismo en esta tarea, sobre el retorno financiero de la actividad, el valor de las bases de datos ad-hoc globales y especializadas, el impacto de la MBE en el diseño de la investigación futura y en la exposición de resultados. Dentro de lo pertinente, al tema que nos ocupa, recoge algunos elementos que no por ser de sentido común, los tenemos siempre presentes: los usuarios y audiencias tienen necesidades diferentes, el traslado de la evidencia a la práctica puede ser más complejo que la fase de generar la evidencia, la evidencia aislada es insuficiente para tomar decisiones, estas requieren de un manejo del contexto.

Comentario: Es interesante constatar esta opinión de cultores maduros y acreditados de la $\mathrm{MBE}$, sobre que la evidencia es necesaria, pero insuficiente para tomar decisiones clínicas y de gestión. Las propuestas de innovaciones en una práctica clínica requieren del conocimiento del contexto en que estas se aplicaran, del manejo de los códigos culturales de quienes participaran y del respaldo de todos los involucrados. Esta estrecha y necesaria integración del mejor conocimiento técnico global (evidencia), con elementos de la identidad o 
contexto socio-cultural de la organización local, que convergen cuando ocurre participación y capacitación real y efectiva, es una simbiosis necesaria, incluso en la imitación de buenas experiencias. Esta mirada integradora que no es una quimera personal, esta siendo considerada cada vez con mayor fuerza, en los nuevos modelos de gestión clínica (clinical governance) ${ }^{(19)}$.

\section{Conclusiones}

En resumen, la introducción de una innovación o cambio en una práctica clínica, es un proceso muy complejo y si bien no hay recetas universales, que garanticen el éxito de la iniciativa, un instructivo, una guía clínica o un decreto claramente no bastan. La calidad de la evidencia y eficacia de la innovación, la disponibilidad y organización de los recursos asociados para implementarla, el conocimiento del contexto donde se aplicará, la participación de los involucrados y finalmente la capacitación pertinente, nos parecen a nosotros y también a los investigadores especializados como condiciones definitivamente fundamentales, para lograrlo.

La información y capacitación debe considerar a todos los actores: directivos, gestores, propiciadores de la innovación, equipo de prestadores sanitarios y pacientes. Los directivos, gestores de la iniciativa y los técnicos elaboradores de nuevas guías de práctica clínica, por su responsabilidad, tienen la primera necesidad de capacitación previa. Ellos deberían conocer no solo la evidencia que sustenta las innovaciones, sino el contexto y recursos con que cuentan la generalidad de instituciones donde la guía va a ser aplicada, para realizar oportunamente, los ajustes organizacionales y proporcionar todos los recursos que den coherencia, calidad y viabilidad al producto a difundir y posicionar.

Aun cuando, la calidad, justificación técnica y recursos necesarios para respaldar la innovación, son básicos e imprescindibles para su validación por el equipo sanitario que la implementará, estos no son suficientes para el cambio de conducta, de allí lo importante de la participación efectiva y educación sanitaria continua y ad-hoc de todos los involucrados. La capacitación de los profesionales aun cuando sea percibida por estos, como justificada y necesaria para facilitar nuevas prácticas, requiere de motivación y estimulo para favorecer su eficacia y producto. Los estímulos parten, como ya hemos mencionado, por la seriedad, coherencia, lógica, calidad del contenido y respaldo de recursos para la implementación de la innovación. Luego siguen con la calidad en la entrega de la capacitación y formalidad en la certificación de ella. El diseño y contenido de la capacitación deben ser cuidadosamente planificados considerando audiencias con roles, realidades y necesidades específicas. El modelo docente debe ser idealmente presencial, considerar el refuerzo periódico, ser interactuante entre docentes y alumnos, con trabajo en grupos pequeños, con distintos niveles de desarrollo y con diversidad de instrumentos de aprendizaje.

\section{Referencias}

1. Minsal. Guía clínica, ACV isquémico del adulto. Disponible en URL: http://www.minsal.cl/ici/ guias_clinicas_ges2006/ACV_GCAUGE_2006_version62.pdf 2006. 
2. Galdames D, Espinoza M, Erazo S, Cid L, Chica J, Larrea D. Las guías clínicas del accidente cerebrovascular del adulto iguían a los clínicos? Rev M ed Chile 2006; 134: 1069-170.

3. Fox RD, Bennett NL. Learning and change. implications for continuing medical education. BM J 1998; 316: 466-8.

4. Harden R, Grant J, Buckley G, H art I. BEM E Guide № 1: Best evidence medical education. M edical Teacher 1999; 21: 553-62.

5. Veninga CC, Lagerlov P, Wahlstrom R, M uskova M , Denig P, Berkhof J, et al. Evaluating an educational intervention to improve the treatment of asthma in four European countries. Drug Education Project Group. Am J Respir Crit Care M ed 1999; 160: 1254-62.

6. Hobbs FD, Erhardt L. Acceptance of guideline recommendations and perceived implementation of coronary heart disease prevention among primary care physicians in five European countries: the Reassessing European Attitudes about Cardiovascular Treatment (REACT) survey. Fam Pract 2002; 19: 596-604.

7. Nogales-GaeteJ. M edicina basada en la evidencia. Rev Chil Neuro-Psiquiat. 1999, 37: 5-10 1999; 37: 5-10.

8. Issenberg SB, M CGaghie WC, Petrusa ER, Lee Gordon D, Scalese RJ. Features and uses of highfidelity medical simulations that lead to effective learning: a BEM E systematic review. M edical Teacher 2005; 27: 10-28.

9. Slotnick HB. Physicians' learning strategies. Chest 2000; 118: 18S-23S.

10. Walsh K. H ow to assess your learning needs. J R Soc M ed 2006; 99: 29-31.

11. Cantillon P, Jones R. Does continuing medical education in general practice make a difference? BM J 1999; 318: 1276-9.

12. Shumway JM, Harden RM. AM EE Guide No. 25: The assessment of learning outcomes for the competent and reflective physician. M edical Teacher 2003; 25: 569-84.

13. Little P, Hayes S. Continuing professional development (CPD): GPs' perceptions of post-graduate education-approved (PGEA) meetings and personal professional development plans (PDPS). Fam Pract 2003; 20: 192-8.

14. Mast L. Application of the problem-based learning model for continuing professional education: a continuing medical education program on managed care issues-Part II. Am J M anag Care 1997; 3: 77-82.

15. Smits PB, de Buisonje CD, Verbeek JH, van Dijk FJ, M etz JC, ten Cate OJ. Problem-based learning versus lecture-based learning in postgraduate medical education. Scand J Work Environ Health. 2003; 29: 280-7.

16. Smits PB, Verbeek JH, Nauta M C, Ten Cate TJ, M etz JC, van Dijk FJ. Factors predictive of successful learning in postgraduate medical education. M ed Educ 2004; 38: 758-66.

17. Nogales-Gaete J, Tagle P, Godoy J, Herlein A, Sanchez-Vega J, Ivanovic-Zuvic F, Salinas R. Conflicto de interés: una reflexion impostergable. Panel del comité editorial. Rev Chil N euro-Psiquiat 2004; 42: 9-21.

18. Wolf F. Lessons to be learned from evidence-based medicine: practice and promise of evidencebased medicineand evidence-based education. Medical Teacher 2000; 22: 251-9.

19. Iglesias R. ¿A quénos referimos cuando hablamos de gestión clínica? Inv Clin Farm 2004; 1: 24-34. Disponible en URL: http://www.revistainvestigacion. pfizer.es/pages/conten/artics/pdfs/icf14-3.pdf

\section{Correspondencia:}

Dr. Jorge Nogales-Gaete

jnogales@ctcinternet.cl 